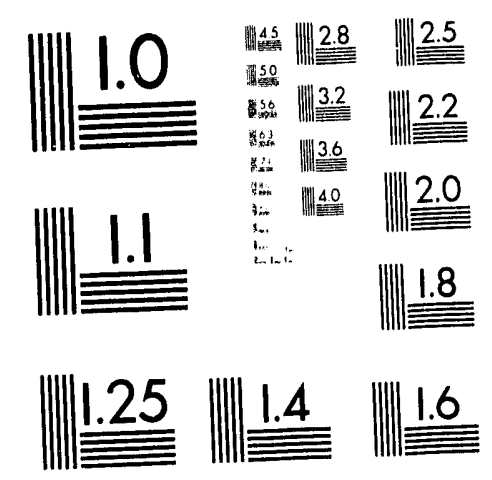



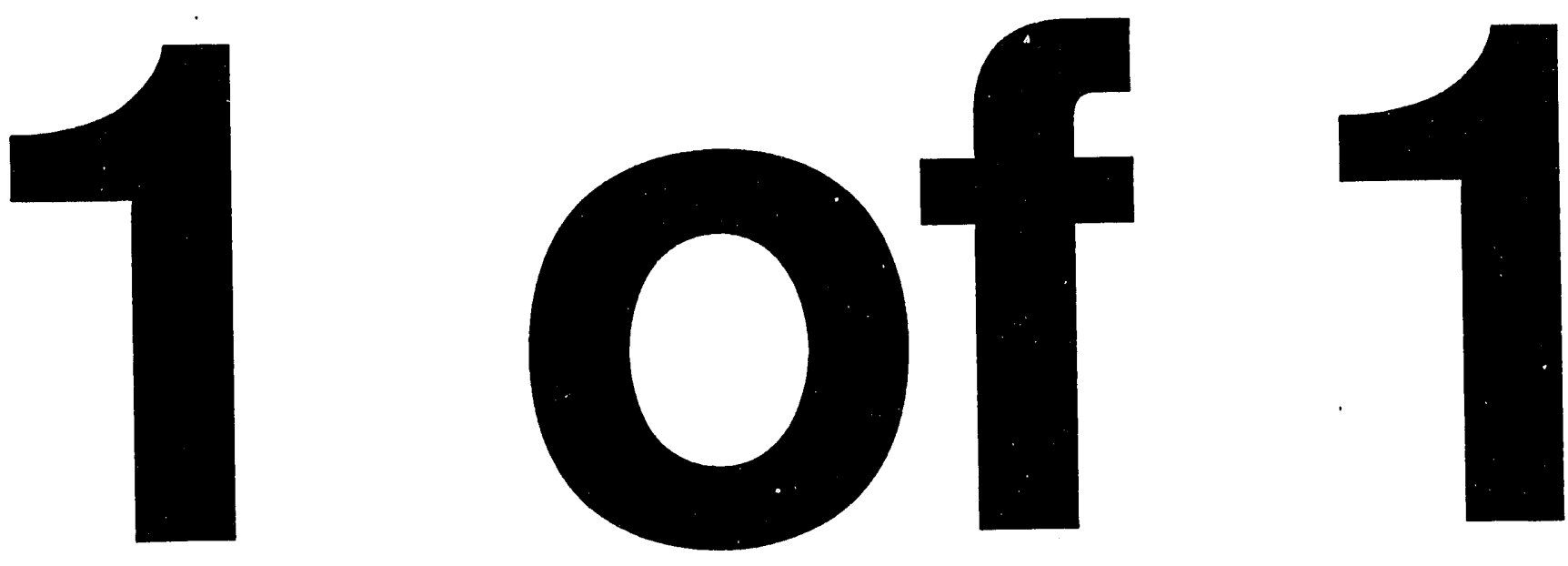


\title{
Characterization of Multiphase Fluid Flow During Air-Sparged Hydrocyclone Flotation by X-Ray CT
}

\author{
Twelfth Quarterly Report \\ 14 May - 13 August, 1993
}

DOE Contract No. DE-FG22-90PC90311

\author{
Submitted by: $\quad$ J. D. Miller \\ Professor of Metallurgical Engineering \\ 216 WBB \\ University of Utah \\ Salt Lake City, Utah 84112 \\ (801) $-581-5160$
}

This report was prepared as an account of work sponsored by an agency of the United States Government. Neither the United States Government nor any agency thereof, nor any of their employees, makes any warranty, express or implied, or assumes any legal liability or responsibility for the accuracy, completeness, or usefulness of any information, apparatus, product, or process disclosed, or represents that its use would not infringe privately owned rights. Reference herein to any specific commercial product, process, or service by trade name, trademark, manufacturer, or otherwise does not necessarily constitute or imply its endorsement, recommendation, or favoring by the United States Government or any agency thereof. The views and opinions of authors expressed herein do not necessarily state or reflect those of the United States Government or any agency thereof.
August 13, 1993 
During this quarter of the DOE project, "Characterization of Multiphase Fluid Flow During Air-Sparged Hydrocyclone Flotation", some experiments from the previous set were repeated to check the reproducibility of the results. Also, a new set of experiments was carried out to study the effects of 1) collector dosage, 2) $Q^{*}$ the nondimensional ratio of air flow rate to slurry flow rate, and 3) slurry pressure on the flow characteristics. Four different $Q^{*}$ values with hydrophobic particles in the feed slurry were studied. The value of $A^{*}$, the dimensionless ratio of overflow opening area to underflow opening area, was maintained at the same level $\left(A^{*}=\right.$ $1.00)$.

Quartz particles of size $-100+200$ mesh were used for this study rather than coal particles because they did not abrade and were of a higher density. The reagents used were 40 ppm (water basis) of MIBC frother and $800 \mathrm{~g}$ of dodecyl amine collector per ton of dry solids in the suspension. In other experiments with no solids in the feed suspension, collector level in the water was varied at 0,10 and $20 \mathrm{ppm}$. At room temperature, quartz is intrinsically hydrophilic while addition of the amine collector renders the quartz particles hydrophobic. The absence of collector will be referred to as the hydrophilic case and the presence of collector will be referred to as the hydrophobic case.

In most cases, a total of 11 scans was taken over the entire length of the ASH unit for each experimental condition. However, for some cases only three sections, at the top, middle and bottom, 
were taken. The previously modified software was used to analyze the CT images obtained from these tests. Some experimental results are presented graphically in Figures 1 through 4 illustrating the reproducibility of the results and the effects of the above mentioned parameters.

Figure $1 \mathrm{~A}$ shows how reproducible these results are. The radial density profiles for identical conditions in two different sets of experiments are presented. It can be seen from this figure that the profiles are very similar in the two cases. The density values compare favorably. The small differences can be attributed to the practical impossibility of maintaining exactly the same conditions in two different sets of experiments on two different days for such a large scale operation. Also, the beam thickness being $2 \mathrm{~mm}$, always contributes an error of $\pm 2 \mathrm{~mm}$. Figure $1 \mathrm{~B}$ shows the radial density profiles for identical conditions but at two different times on the same day. It can be seen from this Figure that there is very little variation in data on the same day. Thus it can be said that the results are fairly reproducible within the limits of experimental error.

Figure 2 shows the effect of collector dosage on flow characteristics in the case of no solids in the feed. It can be seen that the collector level does not significantly influence the flow behavior of the two phase system. However, the general trend reflects that the air core shrinks a little as the collector level is increased from $0 \mathrm{ppm}$ to $20 \mathrm{ppm}$ (water basis). This small change is not detected at the bottom of the ASH.

The effect of $Q^{*}$ on the flow behavior when flotation of 
hydrophobic quartz particles (100 x 200 mesh) is taking place in the ASH, is presented in Figure 3. Examination of Figure 3 reveals that as the $Q^{*}$ value is lowered from 6.83 to 4.55 the air core shrinks. However, below a certain level of $Q^{*}$, the air core dimension becomes independent of the $Q^{*}$ value. The swirl layer thickness is greater in the upper part of the ASH for lower Q* values. Near the bottom, the swirl layer thicknesses become comparable. Note that lower density values are observed for high $Q *$ under which conditions good flotation is achieved if the slurry pressure is adjusted to the correct level. Of course, a higher $Q^{*}$ implies higher air flow rate for a given slurry flow rate. This air helps to achieve better flotation of the hydrophobic particles. Interestingly, the profiles for $Q^{*}=2.28$ and $Q^{*}=1.40$ are identical all along the axis of the ASH. This indicates that the system becomes insensitive to the value of $Q^{*}$ below a threshold level.

The effect of varying slurry pressure on the flow characteristics with actual flotation conditions in the ASH is presented graphically in Figure 4 for two different $Q$ * values. At a higher $Q^{*}$ value $\left(Q^{*}=6.83\right)$, the profiles are similar at the top. But the difference in the shape of the density profiles at the bottom signifies that flotation is better at higher slurry pressure. A near-vertical rise from zero density at the air core to the swirl layer density at the bottom of the ASH implies that the froth phase is narrow there and is depleted of solid particles which results in a lower density in both froth phase and swirl layer. Thus, such a shape of the profile corresponds to better 
flotation of the hydrophobic solid particles to the overflow. In this case the stronger centrifugal force field at a higher slurry pressure leads to better flotation performance. A stronget centrifugal force creates a greater degree of turbulence which helps in achieving a better mixing and particle/bubble contact ard attachment. However, beyond a certain level of turbulence, further increase in the slurry pressure will cause the bubble/particle aggregate to break down and detachment will take place resulting in a decrease in the recovery of hydrophobic particles to the overflow. Available excess air is less for a lower $Q^{*}$ value $\left(Q^{*}=\right.$ 4.55). In this case the decrease in turbulence caused by the lower slurry pressure is not compensated for by the available excess air. Thus the profiles are distinctly different all along the axis of the ASH. Again, the shape of the curves at the bottom implies that recovery of the hydrophobic particles to the overflow is higher for a higher slurry flow rate. Thus, it appears that $Q$ * alone does not define the system. In some cases the slurry pressure must also be taken into account in order to define the system response.

Due to the practical difficulties associated with sampling the product streams at the $\mathrm{x}$-ray $\mathrm{CT}$ site, recovery tests need to be carried out separately. It is intended to perform these tests for several different experimental conditions and determine the flotation response in terms of recovery to the overflow in each case. These responses then will be correlated to the density profiles and hence the flow characteristics.

Based on the $\mathrm{x}$-ray CT testing, work has been initiated towards the development of a phenomenological explanation of the flotation 
response from the established concepts of hindered settling, bubble particle collision/attachment, and bubble/particle slip velocities, etc. 


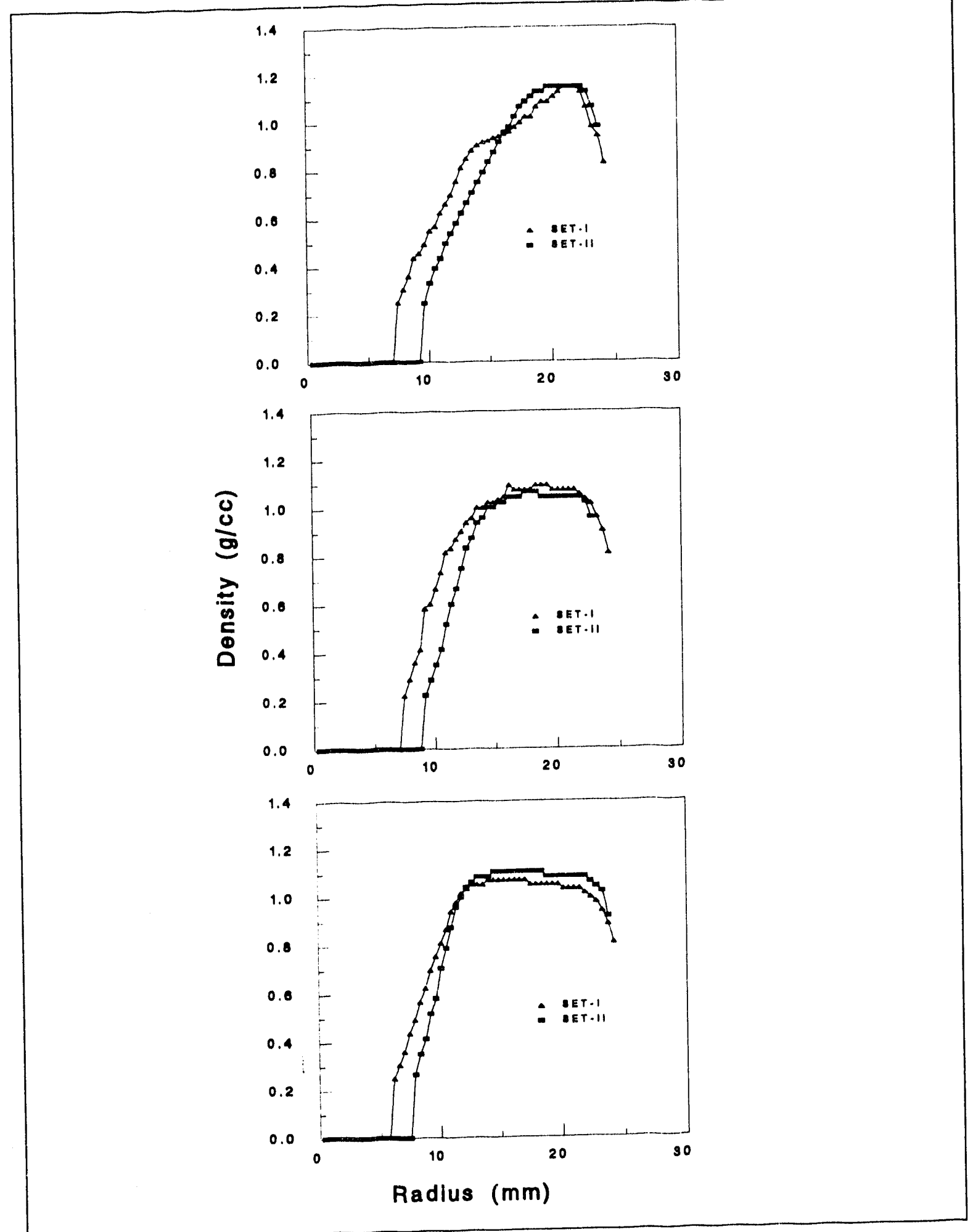

Figure 1A: Radial density profiles at the top, middle and bottom of the ASH for 58 solids, $A^{*}=1.00, Q^{*}=4.55$, hydrophobic particles in two different sets of experiments in order to establish day to day variation in data. 

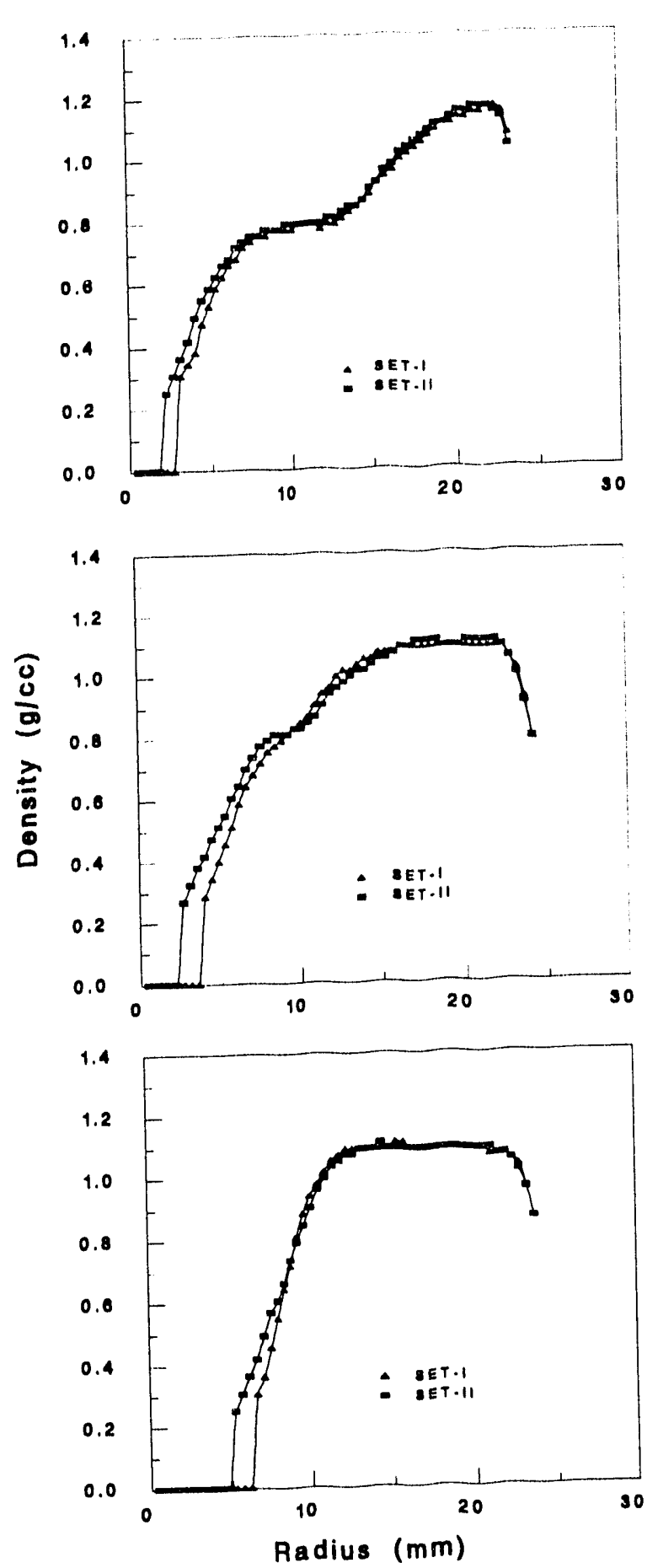

Figure 1B: Radial density profiles for $15 \%$ solids, hydrophobic, $A^{*}=1.00, Q^{*}=4.55$ and 12 psi slurry pressure in two different sets of experiments at two different times in order to establish the variation in data on the same day. 


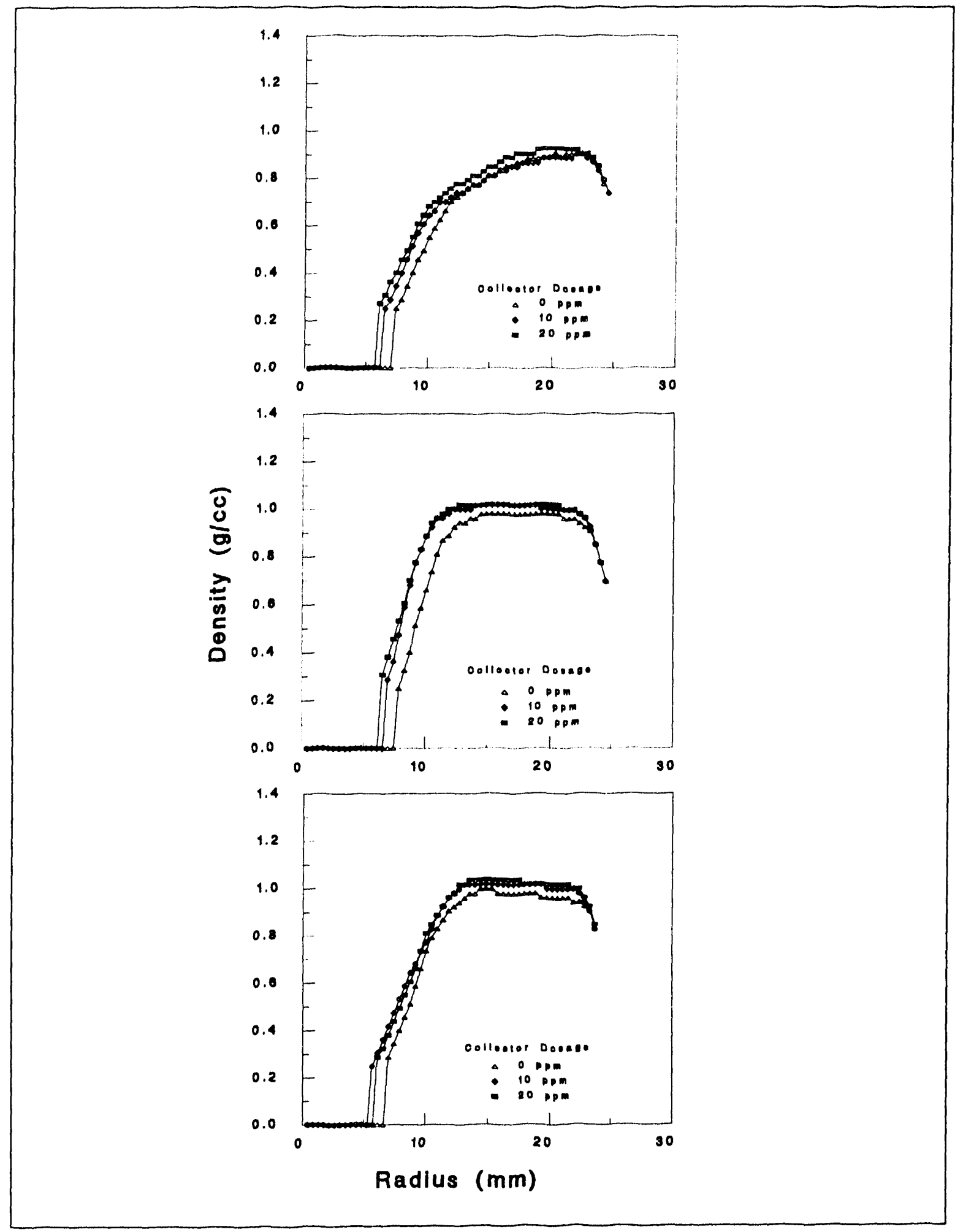

Figure 2: Radial density profile at the top, middle and bottom of the ASH for no solids, 40 ppm frother, $A^{*}=1.00, Q^{*}=4.55$ and 12 psi slurry pressure at different levels of collector (dodecyl amine) addition. 


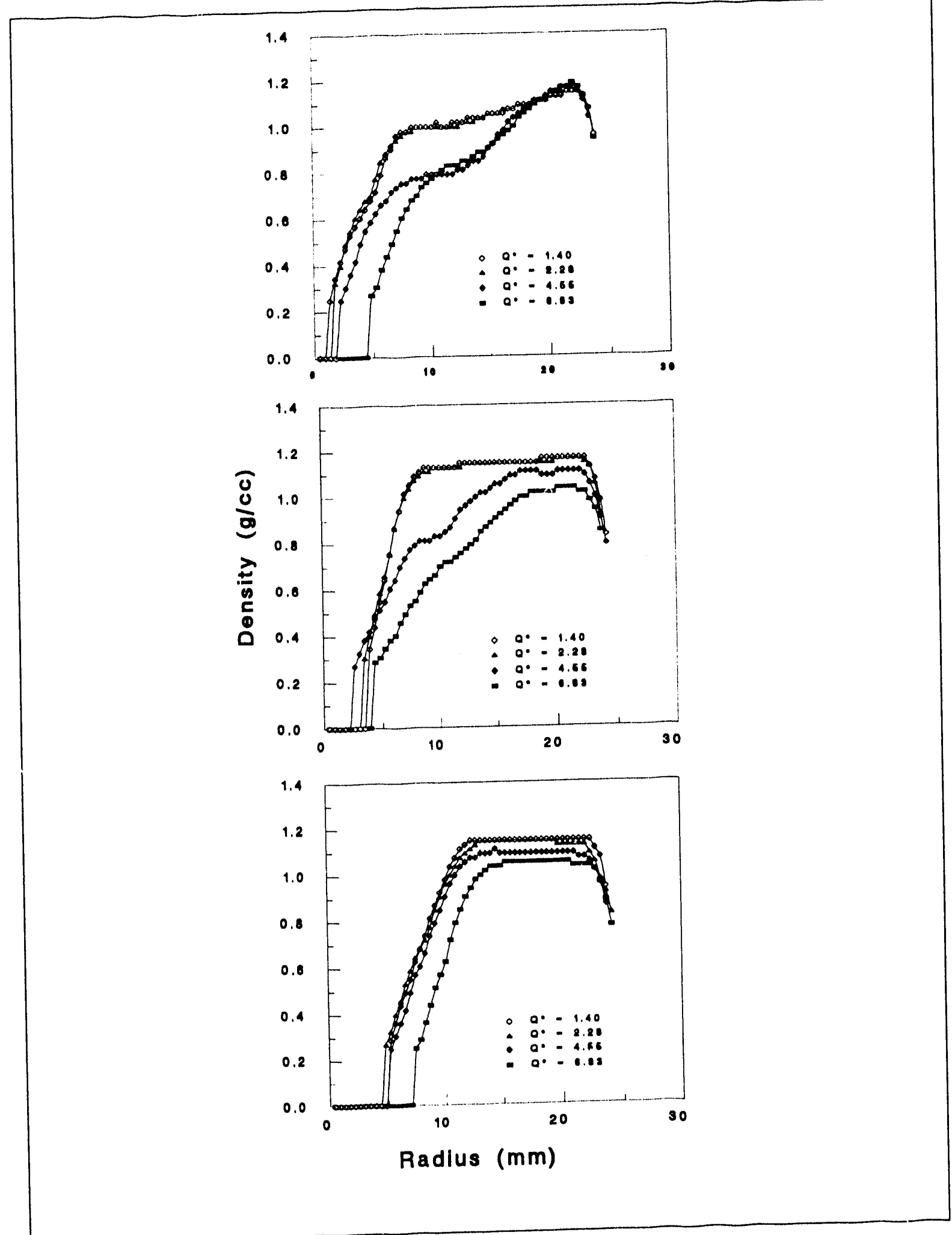

Figure 3: Raidal density profiles at the top, middle and bottom of the ASH for $15 \%$ solids, hydrophobic, $A^{*}=1.00$, and $12 \mathrm{psi}$ slurry pressure at various values of $Q^{\star}$. 


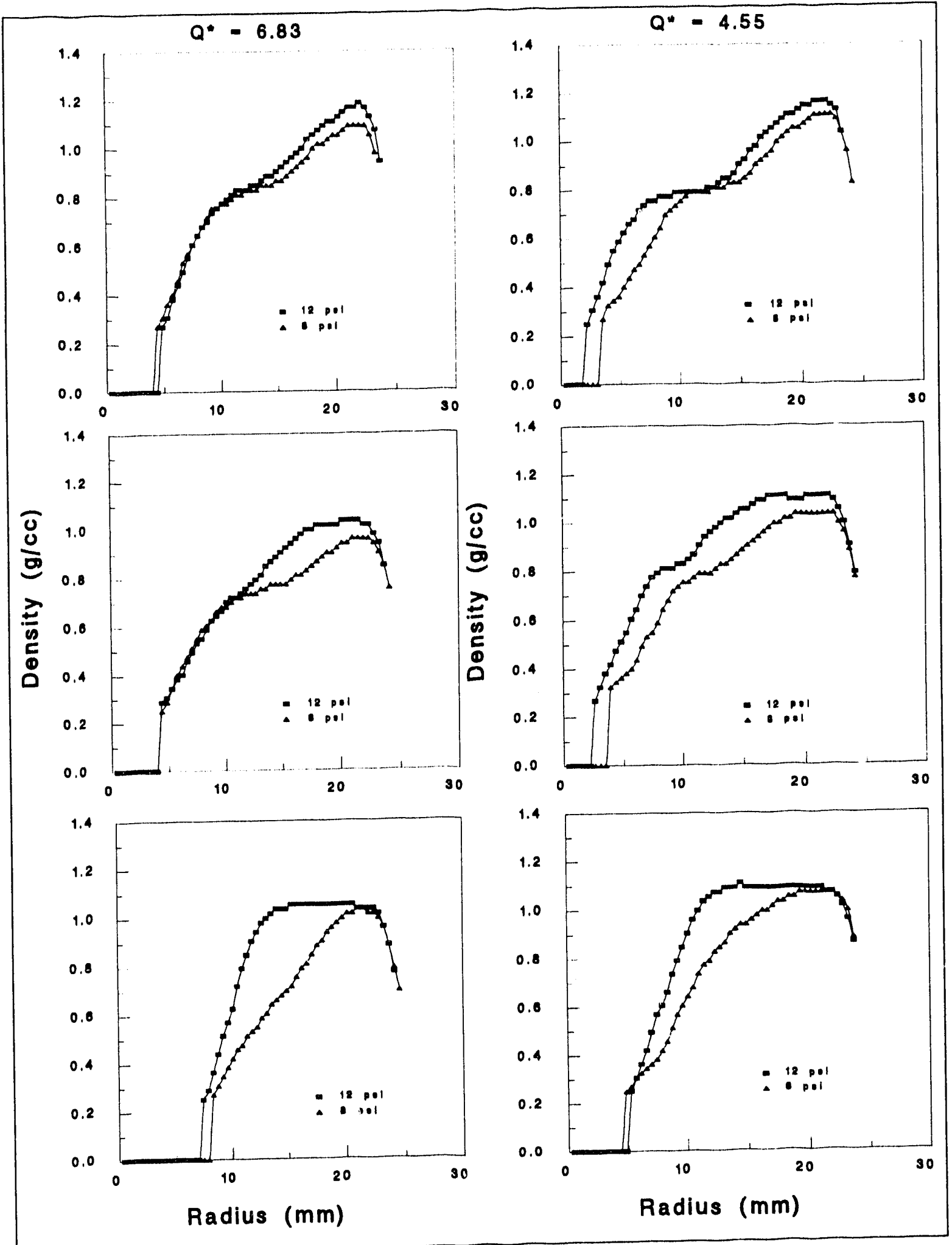

Figure 4: Radial density profiles at the top, middle and bottom of the ASH with $Q^{*}=6.83$ and $Q^{*}=4.55$ for $15 \%$ solids, hydrophobic and $A^{*}=1.00$ for two different slurry pressures. 

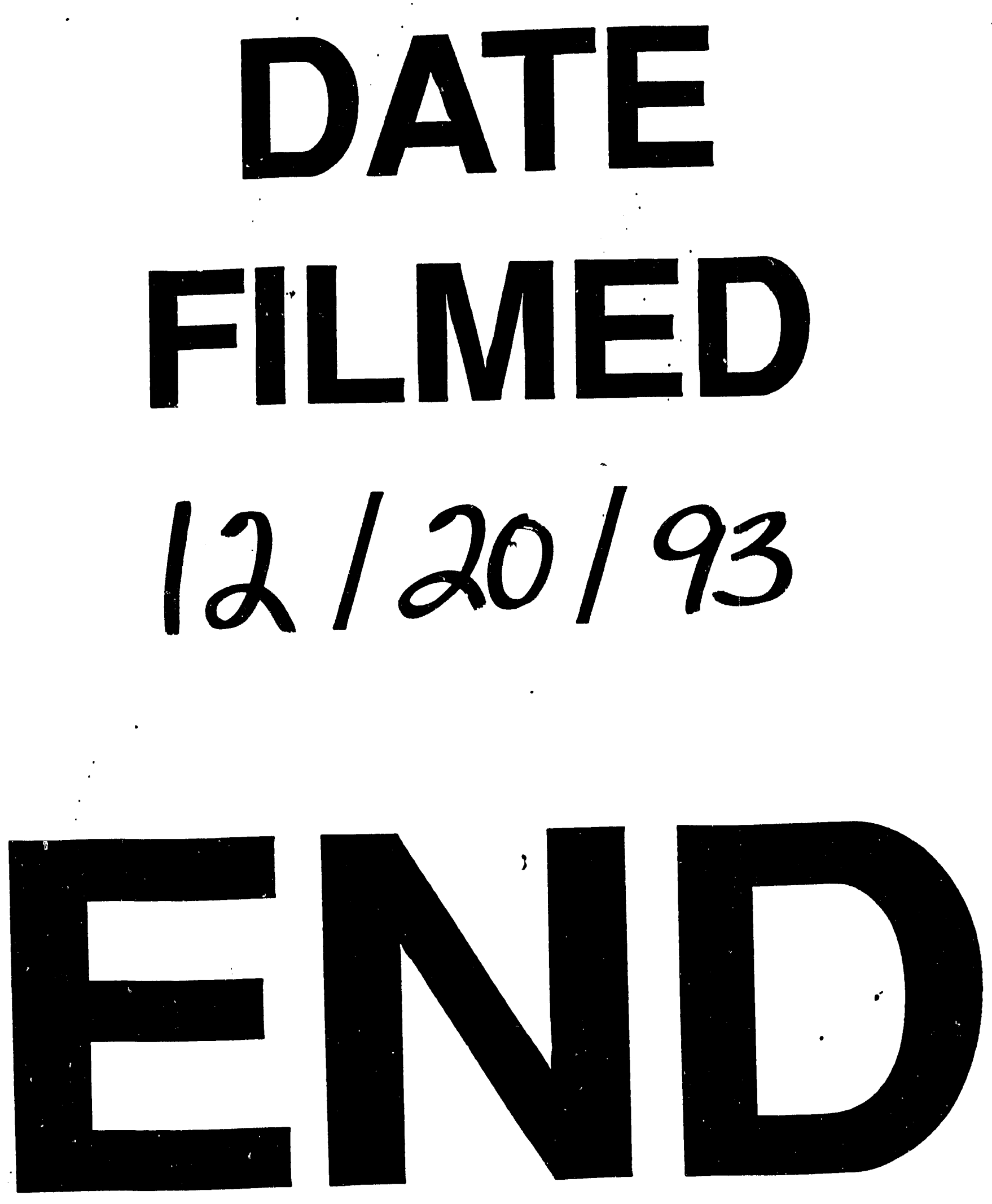
